\title{
1 Learning about larceny: experience can bias bumble bees to secondary-rob nectar
}

3 Jessica L. Barker ${ }^{\mathrm{a}, \mathrm{b} *}$

4 Anna Dornhaus ${ }^{\mathrm{a}}$

5 Judith L. Bronstein ${ }^{\mathrm{a}}$

6 Felicity Muth ${ }^{\mathrm{c}}$

$8 \quad{ }^{a}$ Department of Ecology and Evolutionary Biology, University of Arizona, Tucson, USA

$9 \quad$ b Current address: Aarhus Institute of Advanced Studies, Aarhus University, Aarhus, Denmark

10 'Department of Biology, University of Nevada, Reno, USA

11 *Corresponding author: jbarker@aias.au.dk, +45 87153681

\section{Abstract}

14 How do nectar-feeding animals choose among alternative handling tactics? Such decisions have

15 consequences not only for animal fitness (via food intake) but for plant fitness as well: many

16 animals can choose to 'rob' nectar through holes chewed in the base of a flower instead of

17 'legitimately' collecting it through the flower's opening, thus failing to contact pollen. Although

18 variation among individuals in these nectar-foraging tactics is well documented, it is largely

19 unknown why some individuals specialize (at least in the short term) on robbing, others on

20 legitimate visitation, and others switch behaviors. We investigated whether the frequent tendency

21 to rob nectar through previously constructed holes (secondary robbing) is influenced by prior

22 foraging experience. In a laboratory experiment, we trained groups of bumble bees (Bombus

23 impatiens) either to visit artificial flowers legitimately or to secondary-rob; a third group 
24 received no training. On subsequent visits to flowers, all bees had the opportunity to use either

25 foraging tactic. We found that experience did affect bees' tendency to secondary-rob: trained

26 bees were more likely to adopt the tactic they had previously experienced. Untrained bees

27 initially sampled both tactics, but over time preferred to secondary-rob. Experience also

28 increased bees' success at gaining nectar from flowers, but only for legitimate visitation: that is,

29 experience had a greater effect on the success of the less-preferred tactic. Overall, these findings

30 highlight the importance of experience in animals' choices of alternative handling tactics while

31 foraging, and help explain long-standing observations of variation in nectar-robbing behavior

32 among individuals of the same population.

\section{Significance statement}

35 Animals that can adopt alternative behaviors frequently use only one of them. A widespread but

36 little understood example of this is nectar-feeding, in which foragers can choose between

37 'legitimately' collecting nectar through a flower's opening or 'robbing' nectar via holes chewed

38 through a flower's base. We showed in a laboratory experiment that prior foraging experience

39 can bias bumble bees’ choices to rob nectar from pre-existing holes (i.e., to ‘secondary’ rob).

40 Initial exposure to flowers that could only be robbed or only be legitimately visited increased

41 bees' tendency to subsequently adopt that behavior at the expense of sampling the alternative

42 behavior, even when other bees seemed to prefer the alternative. These results help explain field

43 observations that bees often specialize on a single nectar-collecting behavior and that there is

44 individual variation in which behavior is adopted, contributing to our understanding of nectar-

45 robbing from the animals’ perspective. 


\section{$47 \quad$ Keywords}

48 Nectar robbing, foraging, experience, decision-making, alternative tactics, bumble bee

\section{Introduction}

52 Foraging for floral nectar is widespread, and has important fitness consequences for both animals

53 and plants. The majority of nectar-feeding animals are insects, commonly bees (Hymenoptera),

54 butterflies and moths (Lepidoptera), and flies (Diptera), although other arthropods and many

55 birds, notably hummingbirds, also feed on nectar (Irwin et al. 2010). Since foraging success is

56 known to influence fitness (Schmid-Hempel and Schmid-Hempel 1998; Pelletier and McNeil

57 2003), how an individual manipulates a flower to gain nectar (its nectar-foraging tactic) will

58 likely also impact fitness, because the costs and benefits resulting from different tactics may vary

59 (Pyke 1980; Stephens and Krebs 1987; Gegear and Thomson 2004; Dedej and Delaplane 2005;

60 Stephens et al. 2007). Since nectar-foraging is often associated with pollination, a forager's tactic

61 can also have an impact on plant reproduction (Heinrich and Raven 1972; Goulson 1999; Irwin

62 et al. 2010).

64 Nectar-foraging animals often have choices of alternative tactics for handling flowers. Nectar-

65 foragers typically visit flowers through a flower's opening. This handling tactic is termed

66 'legitimate' because it can result in pollination, yielding reproductive benefits for the plant as

67 well as energetic benefits for the animal (Irwin et al. 2010). However, many animals can also

68 'rob' nectar: they either chew a hole through the flower's corolla ('primary' nectar-robbing), or

69 forage through holes already cut by primary robbers ('secondary' nectar-robbing) (Inouye 1980). 
70 Primary and secondary nectar-robbers usually do not come into contact with a flower's anthers

71 and stigma, and in some species, robbed flowers are subsequently avoided by legitimate visitors.

72 Both robbing behaviors have been extensively documented in a wide variety of plant species

73 (Irwin et al. 2001). Alone or in concert, they usually lead to reduced seed or fruit production

74 (Irwin et al. 2010, Richman et al. 2016); but see Maloof and Inouye 2000; Richardson 2004).

76 While some nectar-foraging species feed on flowers using a single tactic, in others, substantial

77 variation can be observed among individuals: some visit flowers purely legitimately, others

78 exclusively rob nectar, while others adopt a mixed strategy, switching between legitimate

79 visitation and one or both types of robbing (Bronstein et al. 2017). Although the natural history

80 of nectar robbing is well-studied from the plant perspective (including its consequences for plant

81 fitness), the factors affecting animals’ decisions to visit legitimately versus rob nectar remain

82 minimally understood (Irwin et al. 2010; Bronstein et al. 2017). The energetic costs and benefits

83 of each tactic (Dedej and Delaplane 2005) and morphological 'fit' between the nectar-forager

84 and flower likely help explain variation in nectar robbing across species: for example, Bombus

85 species with shorter tongues exhibit more nectar robbing (Newman and Thomson 2005).

86 However, even within species, individuals within a population often vary in nectar robbing (e.g.

87 Apis mellifera: J.L. Barker and J.L. Bronstein, unpubl. data).

89 Since learning plays a major role in determining other aspects of foraging for bumble bees and

90 honey bees, including floral handling strategies (Woodward and Laverty 1992; Laverty 1994a;

91 Chittka and Thomson 1997), it seems plausible that it may also play a role in determining

92 whether or not individuals choose to rob. Previous studies suggest that social learning may be 
93 important. In Bombus terrestris, exposure to the social cue of primary-robbed flowers resulted in

94 a greater likelihood of primary robbing (Leadbeater and Chittka 2008). Bombus lucorum and B.

95 wurflenii appeared to copy other individuals' use of one side of the flower ('handedness') when

96 primary-robbing (Goulson et al. 2013). While these studies show that social experience

97 influences nectar-robbing behavior within species, it is still not clear what role individual

98 foraging experience plays.

99

100 Secondary robbing is ubiquitous in plants subject to primary robbing (Irwin et al. 2010; Richman

101 et al. 2016), yet it is comparatively poorly understood. It is particularly of interest in a behavioral

102 context because flower visitors commonly switch between legitimate foraging and secondary

103 robbing, far more than they do between other foraging tactics (e.g., Richardson and Bronstein

104 2012; Richman 2017). Shifts away from potentially mutualistic, legitimate foraging behaviors

105 towards a nonmutualistic "cheating” tactic has the potential to impact plant success, and indeed 106 to destabilize pollination mutualisms (Bronstein et al. 2017).

108 In this study, we tested whether prior experience with either secondary robbing or legitimately

109 visiting flowers biases individual bumble bees’ decisions to rob nectar. If a bee’s tendency to

110 secondary-rob is not learned (rather, each individual has an a priori tendency to rob or not rob),

111 then a bee’s foraging tactic will not be affected by whether she has had any previous experience

112 with robbing or with legitimate visitation. Alternatively, secondary robbing could arise from

113 previous individual foraging experience: if a bee first encounters opportunities to gain nectar

114 from secondary robbing but not from legitimate visitation, she may continue secondary-robbing

115 flowers over time even if legitimate visitation is subsequently an alternative option that may 
116 yield nectar rewards with equal or even greater success (Ravary et al. 2007). Finally, it is also

117 possible that bees will initially have a preference for a particular foraging tactic (be it innate or

118 learned from prior experience), but that this will change with subsequent experience of sampling

119 both foraging tactics until the individual arrives upon the choice that more successfully yields

120 higher net payoffs (that is, initial floral encounters do not bias longer-term tactic choices).

121

122 We carried out a laboratory-based foraging experiment using the bumble bee Bombus impatiens.

123 We focused only on secondary robbing because this species has never been observed to chew

124 robbing holes itself (i.e., to primary rob); many bees lack the chewing mandibles required to

125 adopt this behavior. This species is, however, well-documented to secondary-rob previously

126 robbed flowers in the field (Rust 1979; Irwin et al. 2010). We trained naïve bumble bees to

127 collect nectar from artificial flowers which they could either only visit legitimately or only

128 secondary-rob; a third group of bees received no training (Fig. 1). This initial training period was

129 intended to capture how, in the field, individuals may gain experience via stochastic initial

130 encounters with flowers in a given environment (for example, due to high variability in the rates

131 of robbed flowers: Irwin and Maloof 2002), as well as via information learned in that

132 environment over time. We then recorded the behavior of these three experimental treatment

133 groups on 'test flowers' that bees could either visit legitimately or secondary-rob over a series of

134 subsequent foraging bouts. This experiment provides insight into how experience via both initial

135 encounters and learning affect tactic choice, but also how experience interacts with tactic type:

136 that is, whether one tactic requires more experience to gain the same rewards. 
140 Subjects and rearing

142 We used four commercially-reared Bombus impatiens colonies (Koppert Biological Systems,

143 Howell, Michigan). We kept two colonies in the laboratory at a given time (colonies A and B:

144 October-November 2013; colonies C and D: March-May 2014), and marked workers every few 145 days with honey bee queen tags (Bee Works, Orillia, Ontario) affixed with superglue, enamel 146 paint (Testor’s, Vernon Hills, Illinois), or paint pens (Craft Smart, Irving, Texas).

148 We housed each colony in a nestbox (39 x $10.5 \times 23 \mathrm{~cm})$, connected by a transparent plastic tube 149 (2 cm diameter) to a foraging arena $(90 \times 40 \times 60 \mathrm{~cm})$ (Fig. 2). By inserting plastic discs into the 150 tube connecting the nestbox and arena, we controlled individual bees' access to the foraging 151 arena. The nestboxes and foraging arenas were made of wood, with transparent Perspex roofs for 152 observation. We placed a sucrose feeder (containing 25\% sucrose in water by volume (v/v)) in 153 each colony’s foraging arena for approximately 1 week, to familiarize the bees with foraging in 154 the arena. We subsequently fed each colony by pipetting $25 \% \mathrm{v} / \mathrm{v}$ sucrose solution into all empty 155 honeypots twice daily, and kept replenished an ad lib. supply of pollen (honey bee-collected, 156 purchased freshly-frozen from Koppert Biological Systems, defrosted and ground before feeding 157 to bumble bees) in a dish next to the nest in the nestbox.

159 Artificial flowers 
161 We constructed artificial flowers out of $0.25 \mathrm{~cm}$ thick yellow foam glued to make a cone $(3.5 \mathrm{~cm}$

162 high x $3.5 \mathrm{~cm}$ diameter), with a $0.5 \mathrm{~cm}$ cut Eppendorf tube tip glued to the bottom to hold $20 \mu \mathrm{l}$

163 of 'nectar' (50\% v/v sucrose solution). We used three types of flowers (Figs. 1 and 2):

164 a. Legitimate-visitation training flowers: the top of the cone was open, allowing a bee access to 165 nectar by entering the flower (B. impatiens is not capable of nectar-robbing in the absence of 166 a pre-existing hole, which was not provided in this treatment). We termed this tactic 167 'legitimate visitation’.

168 b. Secondary-robbing training flowers: the top of the cone was closed with an additional circle 169 of yellow foam, but there was a $0.5 \mathrm{~cm}$ hole cut into the flower, $0.5 \mathrm{~cm}$ above the Eppendorf 170 tube. This allowed a bee to access nectar from outside the flower, at its base, by sticking her 171 proboscis and/or head inside the opening. We termed this feeding tactic 'secondary robbing', 172 the holes cut in these flowers representing those that a primary robber would make.

173 c. Test flowers: the flowers were open at the top and had holes cut into the base, allowing a bee 174 access to nectar via both legitimate visitation and secondary robbing. We note that both

175 'legitimate visitation’ and ‘secondary robbing’ are terms defined from the plant's perspective 176 (see Introduction). Recognizing that this terminology is not meaningful for artificial flowers, 177 we use it here for consistency with field studies of nectar robbing. We also note that, in the 178 field, the handling difficulty and nectar yield of each tactic will depend on the plant species 179 and other factors: see Discussion.

General protocol

183 We assigned bees to one of three experimental treatments (Fig. 1): 
184 Treatment 1: legitimate training. Bees $(N=14)$ foraged from legitimate training flowers (training 185 phase) before gaining access to test flowers (test phase).

186 Treatment 2: secondary-robbing training. Bees $(N=16)$ foraged from secondary-robbing training 187 flowers (training phase) before gaining access to test flowers (test phase).

188 Treatment 3: no training. Bees $(N=17)$ had access to test flowers without any prior encounters 189 with either type of training flowers (test phase only; no training phase).

190 In all training and test phases, we presented bees with an array of 10 artificial flowers (Fig. 2).

191 Each flower was mounted with Blue-Tack on a clear plastic tube $(9.5 \mathrm{~cm}$ high x $2.5 \mathrm{~cm}$

192 diameter) that was glued to a piece of plywood (approximately 45 x $30 \mathrm{~cm}$ ). The foraging arena

193 was illuminated by a lamp with an incandescent $60 \mathrm{~W}$ bulb, in a lab with fluorescent lighting. We

194 mounted a video camera on a tripod next to the arena so that we could record bees' behavior in 195 the arena (viewed from above, through the Perspex roof).

197 Training phase

199 For Treatments 1 and 2, we allowed 10-20 bees at a time access to an array of training flowers 200 (either legitimate-training or robbing-training flowers: Fig. 2) in the foraging arena. These bees 201 had foraged from a sucrose feeder in the arena, but were naïve to the artificial flowers. Whenever 202 a bee made a rewarded visit (i.e., obtained nectar) on a flower, we recorded the bee’s 203 color/number tag, and refilled the flower (so that, as far as possible, all training flowers were 204 always full). 
206 We let bees forage from the training flowers until one bee made 10 rewarded visits. These 10

207 visits could be over multiple bouts, with a bout defined as a sequence of visits to flowers made

208 without returning to the colony (i.e., a single foraging trip). When one bee had gained these 10

209 rewards, we did not remove her immediately but instead allowed her to finish her foraging bout

210 and return to the colony by herself. Consequently, some bees that we tested made more than 10

211 rewarded visits. We then removed all other bees from the foraging arena, cleaned the mount for

212 the flowers with isopropyl alcohol to remove any scent marks, and soaked the training flowers in

213 warm water to remove any traces of nectar. Both were dried before re-use.

214

215 Test phase

216

217 Immediately after training, we tested each bee in Treatments 1 and 2 over a series of five

218 foraging bouts. We carried out the tests on individual bees without others present, to ensure there

219 were no social effects on which tactics bees used. We allowed the test bee access to an array of

220 test flowers in the foraging arena; when she finished foraging (usually after around four

221 rewarded flower visits), we allowed her back into the nestbox via the connecting tube, and

222 counted this series of visits as a single test bout. We then waited for the bee to return to the arena

223 for the next foraging bout. Between test bouts, we replaced the test flowers with clean filled

224 ones, keeping the same mount. When the test bee was ready to resume foraging, we allowed her

225 to return to the arena for another bout, with each test bee completing a total of five bouts (and

226 approximately $<10$ minutes between each bout). The five bouts by each bee were always

227 completed on a single day, and on the same day as that bee’s training phase. 
229 For bees in Treatment 3 (no training), we carried out the test phase in exactly the same way as in

230 Treatments 1 and 2, with the exception that in the first bout, we allowed 10-20 bees into the

231 foraging arena and when one bee gained a reward we removed all other bees in the arena,

232 avoiding disturbance to the foraging bee. We did this by catching the other bees with forceps or a

233 small plastic container, and either putting them back into the nestbox directly or letting them

234 back through the connecting tube. Thus, the first bout was similar to the training phase in

235 Treatments 1 and 2, in that every bee first entered the foraging arena with several other bees, but

236 subsequent testing was on individual bees.

237

238 To determine whether foraging bees fully drained the flowers they visited, we measured nectar

239 volumes in a subset of arrays after bees completed their trial, using $2 \mu$ microcapillary tubes

240 (Fisher Scientific). All flowers on which bees gained the nectar reward ('successful visits') were

241 completely depleted of nectar each time (i.e., bees consumed $20 \mu \mathrm{l}$ of nectar), regardless of

242 whether they had been 'legitimately visited' or 'secondary-robbed'.

244 Analyses

246 For each visit in the test phase, we recorded:

247 a. the foraging tactic used by the test bee (legitimate visitation or secondary robbing, as defined 248 above: see Artificial flowers);

249 b. the location of the flower in the foraging array (we did not refill flowers during testing, so a 250 re-visit would be unrewarded unless the first visit was not successful); 
251 c. the times at which the test bee landed on the flower (defined as first contacting the flower

$252 \quad$ with two legs) and left the flower;

253 d. the times at which the test bee started and stopped attempting to obtain nectar (defined as the

254 bee’s head in the legitimate opening or the robbing-hole); and

255 e. whether or not the test bee was successful at gaining the nectar reward (defined as the bee’s

256 head positioned over the nectar for more than 2 seconds, with abdomen pumping).

257 We used data from real-time observations for (a) and (b), and from video recordings for (c), (d)

258 and (e). The videos were scored by observers who were blind to the experimental treatment. In

259 some videos, the test bee’s behavior was partially obscured by the flowers, and thus the sample

260 sizes for (c), (d) and (e) are smaller.

262 We used R version 3.4 (R Core Team 2017) for statistical analyses. After checking that the 263 residuals were normally distributed, we first ran repeated G-tests of goodness-of-fit (using the R 264 packages RVAideMemoire (Hervé 2017) and dplyr (Wickham et al. 2017)) for each treatment, to

265 determine whether bees' choice of tactic (legitimate visitation versus secondary robbing,

266 measured as the proportion of legitimate visits pooled across all five bouts) differed from chance 267 (50\% probability of using each tactic). We also addressed whether treatments differed from each 268 other and whether this changed across foraging bouts, by fitting linear mixed models (LMMs) 269 using the R package nlme (Jose et al. 2017), all of which included the random factors 'bee’ 270 (individual) and 'colony'.

271 a. Choice of foraging tactic (from real-time data): we used the proportion of legitimate visits in 272 each bout as the response variable, with experimental treatment (legitimate training, 

secondary-robbing training, or no training), bout (1-5), and treatment $\times$ bout interaction as 274 predictor variables.

275 b. Success at obtaining the nectar reward (from video data): we used the proportion of 276 successful visits pooled across all bouts as the response variable, with experimental 277 treatment, tactic type (legitimate visitation or secondary robbing) and treatment $\times$ tactic as $278 \quad$ predictor variables.

279 c. Latency to access nectar the first time (from video data): we considered the first time a bee 280 obtained nectar from a test flower, and defined 'latency' as the time between landing on the 281 flower and beginning to feed. We wanted to know how long it took bees to use each handling 282 tactic, so we used latency to access nectar rather than a measure of foraging efficiency 283 derived from the total time spent on the flower. (We note that the total time on the flower did 284 not differ for the different foraging tactics. Legitimate visits took $22.0 \pm 0.9$ seconds (mean \pm 285 SE), and secondary robbing visits took $23.5 \pm 0.8$ sec.) In our analyses, we used latency as 286 the response variable, with experimental treatment, tactic type, treatment $\times$ tactic as 287 predictors. We analyzed latency on the first visit only because we would expect treatment to 288 have the strongest effect on a bee’s first visit compared to later visits.

289 When we found a significant effect of treatment, we ran Tukey post-hoc tests (using the R 290 package lsmeans) to determine where the differences lay. When there was a significant 291 interaction between treatment and another factor, we ran separate models on subsets of the data 292 to examine the interaction in more detail. 
Prior use of one foraging tactic caused subsequent bias, persisting after opportunities to use

297 both tactics

298

299 Bees showed a bias towards the foraging tactic on which they had been trained (Fig. 3). All

300 trained bees used the tactic on which they had been trained on their first visit to test flowers.

301 Across all bouts, most of the visits made by legitimate-trained bees were legitimate (proportion

302 of legitimate visits: mean \pm standard error (SE): $0.88 \pm 0.03, N=66$ bouts by 14 bees; repeated $G$ -

303 test of goodness-of-fit against expected 0.5: $G_{14}=264, p<0.001$ ), while most of the visits made by

304 secondary-robbing-trained bees were secondary robs (mean \pm SE: $0.16 \pm 0.03, N=77$ bouts by 15

305 bees; $\left.G_{15}=245, p<0.001\right)$. Untrained bees showed no bias towards either tactic on their first visit,

306 but over the five bouts less than $50 \%$ of their visits were legitimate visits (mean \pm SE: $0.32 \pm$

$307 \quad 0.04, N=76$ bouts by 17 bees; $\left.G_{17}=126, p<0.001\right)$.

309 In addition to these departures from random choice, the behavior of bees in the three treatment

310 groups both differed from each other and changed across the foraging bouts (Fig. 3). Legitimate-

311 trained bees made a significantly higher proportion of legitimate visits across the five foraging

312 bouts of the test phase than did secondary-robbing-trained and untrained bees, which did not

313 differ from each other (linear mixed model (LMM) effect of treatment: $F_{2,41}=36.4, p<0.001$;

314 differences confirmed with Tukey post-hoc tests: legitimate-trained versus secondary-robbing-

315 trained bees; $t_{41}=10.8, p<0.001$; legitimate-trained versus untrained: $t_{41}=7.37, p<0.001$;

316 secondary-robbing-trained versus untrained: $t_{41}=1.38, p=0.362$ ). 
318 Untrained bees increased their frequency of robbing over the five bouts, but this was not the case

319 for bees in the two training groups (significant treatment $\times$ bout interaction $\left(\mathrm{LMM}: F_{8,160}=2.14\right.$,

$320 p=0.035$; Fig. 3). Rather, the trained bees persisted with using the tactic on which they had been

321 trained, even after multiple foraging bouts in which they could sample both tactics. To determine

322 how choices changed across bouts for each treatment, we examined the effect of bout in each

323 treatment separately. Bout had a significant effect on untrained bees (LMM: $F_{4,55}=2.68$,

$324 p=0.041)$, but not on bees with training in either legitimate visitation $\left(F_{4,48}=1.49, p=0.220\right)$ or

325 secondary robbing $\left(F_{4,57}=0.858, p=0.494\right)$.

326

327 Prior use of either tactic increased success obtaining nectar

328

329 In order to determine whether in the context of this experiment one tactic might have been more

330 profitable than the other, we analyzed how tactic choices were related to bees' success obtaining

331 nectar. Even without training, bees were highly successful at gaining nectar from flowers: bees in

332 the no-training experimental treatment obtained the nectar reward in $80 \pm 11 \%$ (mean \pm SE) of

333 legitimate visits ( $N=97$ visits by 12 bees) and in $90 \pm 8 \%$ of secondary-robbing visits $(N=177$

334 visits by 13 bees).

335

336 However, training increased bees’ success at gaining nectar from flowers still further (Fig. 4).

337 Bees that were trained in either legitimate visitation or secondary robbing were more successful

338 gaining nectar on legitimate visits than were untrained bees (LMM effect of treatment:

$\left.339 F_{2,31}=17.0, p<0.001\right)$. Untrained bees were more successful gaining nectar when secondary-

340 robbing than when legitimately visiting flowers (LMM treatment $\times$ tactic interaction: $F_{2,27}=9.36$, 
$341 \quad p<0.001$; differences confirmed with Tukey post-hoc tests: for legitimate visits, legitimate-

342 trained versus secondary-robbing-trained bees: $t_{27}=0.720, p=0.754$; legitimate-trained versus

343 untrained: $t_{27}=3.88, p=0.002$; secondary-robbing-trained versus untrained: $t_{27}=3.70, p=0.003$ ).

345 We further investigated the treatment $\times$ tactic interaction by running separate LMMs for each

346 tactic type. We found that bees' training had a stronger effect on their success when visiting

347 legitimately than when secondary robbing. For legitimate visits, treatment significantly affected

348 bees' success $\left(F_{2,28}=10.6, p<0.001\right)$ : bees with no training were less successful at obtaining

349 nectar legitimately than were legitimate-trained and secondary-robbing-trained bees. However,

350 bees' success at secondary robbing was not affected by treatment $\left(F_{2,27}=2.43, p=0.107\right)$.

352 Latency to obtain nectar on the first rewarded visit was not affected either by training (LMM:

$\left.353 F_{2,30}=0.668, p=0.520\right)$ or by the foraging tactic used on the first visit $\left(F_{1,27}=1.13, p=0.296\right)$ (Fig.

354 5).

355

356 Discussion

357

358 Initial floral encounters can bias bees to secondary-rob or not rob nectar

359

360 When do bees and other nectar-foraging animals secondary-rob floral nectar, rather than visiting

361 flowers legitimately, when they have the option of gaining nectar from both tactics? Two long-

362 standing observations from the field remain unexplained. First, bees are often observed using a

363 single nectar-foraging tactic during a single foraging bout, even though they are capable of 
364 employing alternatives (Richardson and Bronstein 2012; Bronstein et al. 2017; J.L. Barker and

365 J.L. Bronstein, unpubl. data). Second, the extent to which individuals rob is observed to vary

366 within a species and even within a population (Irwin et al. 2010; Richardson and Bronstein

367 2012). Our laboratory experiment offers an explanation for both of these field observations: we

368 showed that bumble bees (Bombus impatiens) specialized on secondary-robbing or legitimate

369 visitation depending on which of these tactics they had initially used (Fig. 3). Bees that had not

370 had prior exposure to either tactic did not show an initial preference. Thus, secondary nectar

371 robbing is affected by individual foraging experience.

372

373 Bees that had prior experience collecting nectar from our artificial flowers did not change their

374 tactic choices over the subsequent five foraging bouts of the experiment, instead continuing to

375 predominantly adopt the tactic they had already used (Fig. 3). In contrast, bees that had not

376 previously encountered the artificial flowers did change their tactic choice over the course of the

377 experiment. These untrained bees began by using both tactics, but were less likely to visit

378 flowers legitimately over time, such that in later bouts they secondary-robbed at the same

379 frequency as bees trained to do so (Fig. 3), implying that secondary robbing may be the optimal

380 tactic on these artificial flowers. Together, the results indicate that although bees can sample

381 alternative tactics and may eventually learn to use the more profitable one, repeated initial

382 opportunities to employ one tactic can persistently bias bees’ later choices. A possible

383 explanation is that using a single handling tactic over the course of several bouts means that

384 alternative tactics do not need to be stored in a bee's short-term memory (reviewed by Bronstein

385 et al. 2017). Although insects are known to employ a broad range of cognitive abilities when

386 visiting flowers (Chittka and Thomson 2005), this is, to our knowledge, the first evidence that 
387 individual experience plays a role in determining whether individuals secondary-rob flowers or

388 visit them legitimately.

390 Why might a bias based on initial encounters be beneficial?

392 Our finding that secondary nectar robbing was affected by the foraging environment to which 393 bumble bees were first exposed is consistent with evidence that prior encounters can result in 394 biased tactic choices in other aspects of nectar foraging, for example 'handedness' in flower395 handling (Kells and Goulson 2001) and constancy to certain flower morphologies (Laverty 396 1994b; Ishii and Kadoya 2016). Floral constancy - the tendency to choose the same flower color 397 or morphology repeatedly despite opportunities to forage from alternative types (Grant 1950;

398 Heinrich 1976; Waser 1986) - is analogous to the decision to adopt a single handling tactic 399 (nectar robbing versus legitimate visitation) on a given flower type (Bronstein et al. 2017). Many 400 hypotheses for floral constancy invoke learning and cognitive mechanisms (Chittka et al. 1999; 401 Chittka and Raine 2006; Chittka and Muller 2009), and can provide insight into the benefits of 402 biases arising from prior encounters. Given that a tendency to bypass rewarding alternatives can 403 be costly (Costa et al. 2016), when might such biases be beneficial?

405 First, since acquiring information about alternatives may be costly, it frequently pays to use a 406 tactic that was previously successful. Bumble bees are less likely to switch flower morphotype 407 when the environment is less variable, as the available tactics and their net benefits are also less 408 variable (Chittka et al. 1997; Gegear and Thomson 2004; Keasar et al. 2013). Thus, during the 409 short lifespan of a bumble bee worker, adopting a single foraging tactic that provides acceptable 
410 nectar rewards may yield a higher expected net benefit than sampling many other tactics that are

411 costly to acquire even if potentially more rewarding. Second, there may be gains from efficiency

412 (Laverty 1980, 1994b; Lewis 1986; Keasar et al. 1996). Bumble bees switching between

413 foraging tactics that require different motor patterns incur costs of increased memory load,

414 handling time, and time flying between flowers (Chittka et al. 1999).

The effect of bee biases on plant pollination traits

418 On the artificial flowers we used, secondary nectar robbing was apparently preferred over

419 legitimate visitation because it was the 'easier' tactic. Three results lead to this conclusion. First, 420 bees that were not trained for a particular tactic preferred secondary-robbing as they gained 421 experience with both tactics (Fig. 3): bumble bees prefer flowers that require simpler handling 422 and less learning unless morphologically complex flowers are much more rewarding (Muth et al. 423 2015). Second, untrained bees in our experiment were more successful obtaining nectar via 424 secondary robbing than legitimate visitation (Fig. 4). Third, training via prior use of either tactic 425 increased bees’ success at legitimate visits, but not at secondary robbing: learning has been 426 shown to disproportionately improve performance at more 'difficult' handling tactics (Heinrich 427 1979; Laverty 1980).

429 While bees have been shown to prefer secondary robbing over legitimate visitation on certain 430 flower morphotypes in nature (Free 1968; Dedej and Delaplane 2005), it is unknown whether 431 this is more generally the case. Bees are likely to profit from a secondary-robbing tactic only at 432 times and places when primary-robbing rates are high; this is a tactic that can only be used in the 
433 presence of primary-robbing species, and is likely only profitable once robbing-holes are

434 abundant (Richman et al. 2017; J.L. Barker and J.L. Bronstein, unpubl. data) and when nectar

435 has not been greatly depleted by primary robbing. The extent to which bees switch from

436 legitimate visitation to secondary nectar robbing could also be influenced by specific features of

437 different flowers and bees' interactions with them. For example, bees’ choices of different flower

438 morphotypes depended on whether their previous experience was successful (Chittka et al. 1997)

439 and whether they encountered a given morphotype consecutively or mixed in with other flowers

440 (Chittka and Thomson 1997). As different morphotypes often require different handling tactics,

441 it is probable that these factors also affect bees' choices of handling tactics with regard to nectar

442 robbing. An additional consideration to account for when bees may switch nectar-foraging

443 tactics is whether those bees are also foraging for pollen on the same species. Because secondary

444 robbing bypasses a flower’s anthers, bees generally pick up little to no pollen per visit. Thus,

445 pollen-collecting bees may be less likely to switch to secondary robbing than those exclusively

446 foraging for nectar.

448 Plants are likely to gain greater fitness benefits when their floral visitors specialize on legitimate

449 visits, as legitimate visitation generally transfers pollen and nectar robbing does not (Irwin et al.

450 2001). How, then, might plants be able to increase the likelihood of visitor specialization on

451 legitimate visits? While one mechanism might be for the payoff from legitimate visitation to be

452 greater than that from nectar robbing (e.g., plants may be able to reduce the payoff of primary

453 robbing via thickened calyces (Irwin et al. 2010)), our study also suggests that plants may

454 disproportionately decrease secondary robbing if bees’ initial encounters, and success at

455 extracting nectar, can be biased towards legitimate visits. Thus we would might expect selection 
456 for floral traits that particularly affect naïve bees, such as traits like nectar guides that exploit 457 sensory biases (Leonard et al. 2013).

459 Implications

461 Our experiment shows that bumble bees’ initial opportunities to use a given nectar-foraging 462 tactic affected their subsequent tactic choice and success. We suggest that this result can help 463 explain why, in nature, some individuals rob nectar while others visit flowers legitimately or 464 adopt mixed strategies (reviewed by Irwin et al. 2010; Bronstein et al. 2017). In the field, the 465 frequency of flowers that can be secondary-robbed varies both spatially and temporally (Irwin 466 and Maloof 2002). Individual bumble bees will likely encounter different floral environments 467 depending on where and when they first leave the nest to begin foraging (Heinrich 1976).

468 Consequently, if a naïve bumble bee started foraging from flowers before any of those flowers 469 had been primary-robbed by other floral visitors (thus precluding secondary robbing), then she 470 might be 'primed' by that environment to continue visiting flowers legitimately. However, our 471 experiment addressed a relatively short time scale (five foraging bouts in a single day) and a 472 fixed environment; field observations of honey bees suggest that they learn to secondary-rob as 473 the frequency of primary-robbed flowers increases (Dedej and Delaplane 2004).

475 The artificial flowers we used likely required more similar handling techniques for both 476 legitimate visitation and secondary robbing than is likely for real flowers, since in both cases 477 bees merely had to find an opening. Similar handling techniques can enable transfer of motor 478 skills (Dukas 1995; Chittka and Muller 2009), explaining why prior encounters with either type 
479 of flower in our experiment (providing rewards only via legitimate visitation or only via

480 secondary robbing), increased bees' success at legitimate visits compared to bees that had no

481 prior encounters with any flowers (Fig. 4). The artificial flowers’ morphology may also explain

482 why bees’ prior encounters did not affect their latency to find nectar on the first visit where they

483 did find so (Fig. 5): the nectar reward was relatively straightforward to access via both handling

484 tactics. In addition, each handling tactic on our flowers yielded the same volume and

485 concentration of nectar. Whether this is the case in nature, and how differences in the nectar

486 reward affect bees’ behavioral flexibility, is an open question. Robbed flowers commonly

487 contain less nectar, on average, than unrobbed flowers (Irwin et al. 2010), so bees that previously

488 fed legitimately from unrobbed flowers may be more likely to switch to secondary-robbing on

489 robbed flowers if continued legitimate visits result in decreased nectar returns. Secondary

490 robbing may also allow bees to extract the last dregs of nectar more effectively (Dedej and

491 Delaplane 2005).

492

493 In sum, we found that biases in bees' choice of nectar-foraging tactics arose from initial

494 encounters with opportunities to use each tactic, and, within the time-scale and relative nectar

495 rewards afforded by our experiment, were not overcome by subsequent exposure to additional

496 tactic options of different 'preferability'. These results should apply broadly to the many

497 situations where animals choose between alternative handling tactics for the same food sources

498 (Custance et al. 1999; Aplin et al. 2013; Mondal et al. 2014). Thus, our study not only helps

499 explain behavioral flexibility in nectar robbing, but also sheds light on the range of mechanisms

500 by which biased tactic choices can arise. 


\section{Acknowledgements}

503 This work was funded by National Institutes of Health grant 5K12GM000708-13; National

504 Science Foundation grants DEB-1262292, DEB-1354155, IOS-0921280, IOS-1455983; and the

505 Aarhus Institute of Advanced Studies - Marie Curie COFUND Fellowship. We thank Dan Papaj

506 and Caitlin Stern for insightful discussion, and the Bronstein and Papaj labs at the University of

507 Arizona for loan of equipment and further helpful discussion. Kit Henker, Autri Hafezi, Kellen

508 Fortier, Gianna Trujillo, Nico Rizzo, Daniel Thews, Ellen Young, Sage Cutler and Michael

509 Rivera assisted with conducting experiments; Heather Ellison, Leyton Galapia, Laker Dohan and

510 Nico Rizzo assisted with scoring videos.

511

\section{$512 \quad$ Ethical approval}

513 All applicable international, national and institutional guidelines for the care and use of animals

514 were followed.

515

516 Conflicts of interest

517 The authors declare that they have no conflicts of interest.

518

519 Data availability

520 Upon acceptance, data will be archived at Dryad or Behavioral Ecology and Sociobiology’s

521 preferred data depository. We have included our data in this submission as a supplementary

522 Excel file.

523

524 References 
525 Aplin LM, Sheldon BC, Morand-Ferron J (2013) Milk bottles revisited: social learning and

526 individual variation in the blue tit, Cyanistes caeruleus. Anim Behav 85:1225-1232. doi:

527 10.1016/j.anbehav.2013.03.009

528 Bronstein JL, Barker JL, Lichtenberg EM, et al (2017) The behavioral ecology of nectar robbing:

529 why be tactic constant? Curr Opin Insect Sci 21:14-18. doi: 10.1016/j.cois.2017.05.013

530 Chittka L, Gumbert A, Kunze J (1997) Foraging dynamics of bumble bees: correlates of

531 movements within and between plant species. Behav Ecol 8:239-249. doi:

532 10.1093/beheco/8.3.239

533 Chittka L, Muller H (2009) Learning, specialization, efficiency and task allocation in social 534 insects. Commun Integr Biol 2:151-154. doi: 10.4161/cib.7600

535 Chittka L, Raine NE (2006) Recognition of flowers by pollinators. Curr Opin Plant Biol 9:428536 435. doi: 10.1016/j.pbi.2006.05.002

537 Chittka L, Thomson JD (1997) Sensori-motor learning and its relevance for task specialization in 538 bumble bees. Behav Ecol Sociobiol 41:385-398. doi: 10.1007/s002650050400

539 Chittka L, Thomson JD (eds) (2005) Cognitive Ecology of Pollination: Animal Behaviour and $540 \quad$ Floral Evolution. Cambridge University Press, Cambridge

541 Chittka L, Thomson JD, Waser NM (1999) Flower constancy, insect psychology, and plant 542 evolution. Naturwissenschaften 86:361-377. doi: 10.1007/s001140050636

543 Costa TM, Hebets EA, Melo D, Willemart RH (2016) Costly learning: preference for familiar 544 food persists despite negative impact on survival. Biol Lett 12:20160256. doi:

$545 \quad 10.1098 /$ rsbl.2016.0256

546 Custance D, Whiten A, Fredman T (1999) Social learning of an artificial fruit task in capuchin 

monkeys (Cebus apella). J Comp Psychol 113:13-23. doi: 10.1037/0735-7036.113.1.13

Dedej S, Delaplane KS (2005) Net energetic advantage drives honey bees (Apis mellifera L) to nectar larceny in Vaccinium ashei Reade. Behav Ecol Sociobiol 57:398-403. doi:

$$
\text { 10.1007/s00265-004-0852-z }
$$

Dedej S, Delaplane KS (2004) Nectar-robbing carpenter bees reduce seed-setting capability of honey bees (Hymenoptera: Apidae) in rabbiteye blueberry, Vaccinium ashei, "Climax.” Environ Entomol 33:100-106. doi: 10.1603/0046-225X-33.1.100

Dukas R (1995) Transfer and interference in bumblebee learning. Anim Behav 49:1481-1490. doi: 10.1016/0003-3472(95)90069-1

Free JB (1968) The behaviour of bees visiting runner beans (Phaseolus multiflorus). J Appl Ecol 5:631-638. doi: 10.2307/2401637

Gegear RJ, Thomson JD (2004) Does the flower constancy of bumble bees reflect foraging economics? Ethology 110:793-805. doi: 10.1111/j.1439-0310.2004.01010.x

Goulson D (1999) Foraging strategies of insects for gathering nectar and pollen, and implications for plant ecology and evolution. Perspect Plant Ecol Evol Syst 2:185-209. doi: 10.1078/1433-8319-00070

Goulson D, Park KJ, Tinsley MC, et al (2013) Social learning drives handedness in nectarrobbing bumblebees. Behav Ecol Sociobiol. doi: 10.1007/s00265-013-1539-0

Grant V (1950) The flower constancy of bees. Bot Rev 16:379-398. doi: 10.1007/BF02869992

Heinrich B (1976) The foraging specializations of individual bumblebees. Ecol Monogr 46:105128. doi: $10.2307 / 1942246$

Heinrich B (1979) “Majoring” and “minoring” by foraging bumblebees, Bombus vagans: an 

experimental analysis. Ecology 60:245-255. doi: 10.2307/1937652

570 571

572 573 574 575 576

Heinrich B, Raven PH (1972) Energetics and pollination ecology. Science 176:597-602. doi: 10.1126/science.176.4035.597

Hervé M (2017) RVAideMemoire: Diverse basic statistical and graphical functions.

Inouye DW (1980) The terminology of floral larceny. Ecology 61:1251-1253. doi: $10.2307 / 1936841$

Irwin RE, Brody AK, Waser NM (2001) The impact of floral larceny on individuals, populations, and communities. Oecologia 129:161-168. doi: 10.1007/s004420100739

Irwin RE, Bronstein JL, Manson JS, Richardson L (2010) Nectar robbing: ecological and evolutionary perspectives. Annu Rev Ecol Evol Syst 41:271-292. doi: 10.1146/annurev.ecolsys.110308.120330

Irwin RE, Maloof JE (2002) Variation in nectar robbing over time, space, and species. Oecologia 133:525-533. doi: 10.1007/s00442-002-1060-z

Ishii HS, Kadoya EZ (2016) Legitimate visitors and nectar robbers on Trifolium pratense showed contrasting flower fidelity versus co-flowering plant species: could motor learning be a major determinant of flower constancy by bumble bees? Behav Ecol Sociobiol 70:377-386. doi: 10.1007/s00265-016-2057-7

Jose P, Douglas B, Saikat D, et al (2017) nlme: Linear and nonlinear mixed effects models.

Keasar T, Motro U, Shmida A (2013) Temporal reward variability promotes sampling of a new flower type by bumblebees. Anim Behav 86:747-753. doi: 10.1016/j.anbehav.2013.07.010

Keasar T, Motro U, Shur Y, Shmida A (1996) Overnight memory retention of foraging skills by bumblebees is imperfect. Anim Behav 52:95-104. doi: 10.1006/anbe.1996.0155 
591 Kells AR, Goulson D (2001) Evidence for handedness in bumblebees. J Insect Behav 14:47-55.

592 doi: 10.1023/A:1007897512570

593 Laverty TM (1994a) Costs to foraging bumble bees of switching plant species. Can J Zool

594 72:43-47. doi: 10.1139/z94-007

595 Laverty TM (1994b) Bumble bee learning and flower morphology. Anim Behav 47:531-545.

596 doi: 10.1006/anbe.1994.1077

597 Laverty TM (1980) The flower-visiting behaviour of bumble bees: floral complexity and

598 learning. Can J Zool 58:1324-1335. doi: 10.1139/z80-184

599 Leadbeater E, Chittka L (2008) Social transmission of nectar-robbing behaviour in bumble-bees.

600 Proc R Soc B 275:1669-1674. doi: 10.1098/rspb.2008.0270

601 Leonard AS, Brent J, Papaj DR, Dornhaus A (2013) Floral nectar guide patterns discourage

602 nectar robbing by bumble bees. PLoS One 8:e55914. doi: 10.1371/journal.pone.0055914

603 Lewis AC (1986) Memory constraints and flower choice in Pieris rapae. Science 232:863-865.

604 doi: 10.1126/science.232.4752.863

605 Maloof JE, Inouye DW (2000) Are nectar robbers cheaters or mutualists? Ecology 81:2651-

606 2661. doi: 10. 2307/177331

607 Mondal S, Hutchings JA, Herbert GS (2014) A note on edge drilling predation by naticid

608 gastropods. J Molluscan Stud 80:206-212. doi: 10.1093/mollus/eyu001

609 Muth F, Keasar T, Dornhaus A (2015) Trading off short-term costs for long-term gains: how do

610 bumblebees decide to learn morphologically complex flowers? Anim Behav 101:191-199.

611 doi: 10.1016/j.anbehav.2014.12.024

612 Newman DA, Thomson JD (2005) Effects of nectar robbing on nectar dynamics and bumblebee 
613 foraging strategies in Linaria vulgaris (Scrophulariaceae). Oikos 110:309-320. doi:

$614 \quad$ 10.1111/j.0030-1299.2005.13884.x

615 Pelletier L, McNeil JN (2003) The effect of food supplementation on reproductive success in 616 bumblebee field colonies. Oikos 103:688-694. doi: 10.1034/j.1600-0706.2003.12592.x

617 Pyke GH (1980) Optimal foraging in bumblebees: calculation of net rate of energy intake and 618 optimal patch choice. Theor Popul Biol 17:232-246. doi: 10.1016/0040-5809(80)90008-8

619 R Core Team (2017) R: A language and environment for statistical computing.

620 Ravary F, Lecoutey E, Kaminski G, et al (2007) Individual experience alone can generate lasting 621 division of labor in ants. Curr Biol 17:1308-1312. doi: 10.1016/j.cub.2007.06.047

622 Richardson LL, Bronstein JL (2012) Reproductive biology of pointleaf manzanita

623 (Arctostaphylos pungens) and the pollinator-nectar robber spectrum. J Pollinat Ecol 9:115624123.

625 Richardson SC (2004) Are nectar-robbers mutualists or antagonists? Oecologia 139:246-254.

626 doi: 10.1007/s00442-004-1504-8

627 Richman SK, Irwin RE, Nelson CJ, Bronstein JL (2016) Facilitated exploitation of pollination 628 mutualisms: fitness consequences for plants. J Ecol doi: 10.1111/1365-2745.12657.

629 Richman SK, Irwin RE, Bronstein JL (2017) Foraging strategy predicts foraging economy in a 630 facultative secondary nectar robber. Oikos doi: 10.1111/oik.04225.

631 Rust RW (1979) Pollination of Impatiens capensis: pollinators and nectar robbers. J Kansas $632 \quad$ Entomol Soc 52:297-308.

633 Schmid-Hempel R, Schmid-Hempel P (1998) Colony performance and immunocompetence of a 

social insect, Bombus terrestris, in poor and variable environments. Funct Ecol 12:22-30. doi: 10.1046/j.1365-2435.1998.00153.x

Stephens DW, Brown JS, Ydenberg. RC (2007) Foraging: Behavior and Ecology. University of Chicago Press, Chicago

Stephens DW, Krebs JR (1987) Foraging Theory. Princeton University Press, Princeton, NJ

Waser NM (1986) Flower constancy: definition, cause and measurement. Am Nat 127:593-603. doi: $10.1086 / 284507$

641 Wickham H, Francois R, Henry L, Müller K (2017) dplyr: A grammar of data manipulation.

642 Woodward GL, Laverty TM (1992) Recall of flower handling skills by bumble bees: a test of 643 Darwin’s interference hypothesis. Anim Behav 44:1045-1051. doi: 10.1016/S0003$644 \quad 3472(05) 80316-1$ Figure captions

Fig. 1 Treatments 1 and 2 consisted of a training phase and a test phase. In the training phase,

649 bees foraged from artificial flowers that they could either only legitimately visit (flower was

650 open at the top; Treatment 1 ) or only secondary-rob (flower was closed at the top and had a pre-

651 cut hole at the base; Treatment 2). In the test phase, bees were presented with artificial flowers

652 on which they could use both foraging tactics (open at the top plus hole at the base). Treatment 3

653 had no training phase; bees foraged from the test flowers only 
655 Fig. 2 Each colony was housed in a nestbox connected to a foraging arena; a series of discs

656 inserted in the connecting tube allowed us to control individual bees’ access to the arena. Bees

657 foraged on an array of 10 artificial flowers. An array contained one of three types of flowers

658 (legitimate training, secondary-robbing training, or test flowers), depending on the experimental

659 treatment and phase of the experiment

661 Fig. 3 Bees that had been trained on one tactic (legitimate visitation or secondary robbing)

662 predominantly used the trained tactic throughout the experiment, while bees with no training

663 decreased their proportion of legitimate visits over the five foraging bouts. Boxes indicate the

664 quartiles, and whiskers extend to values within 1.5 times the interquartile range. Numbers above

665 the boxes are sample sizes (numbers of bees)

666

667 Fig. 4 Bees’ training did not affect their success obtaining nectar via secondary robbing. For

668 legitimate visits, bees that received no training were less successful than bees that had received

669 either legitimate or secondary-robbing training. Bees with no training were less successful at

670 obtaining nectar legitimately than via secondary robbing. Boxes indicate the quartiles, and

671 whiskers extend to values within 1.5 times the interquartile range. Letters indicate groups that are

672 significantly different, and sample sizes are given below the boxes

673

674 Fig. 5 Training on either foraging tactic (legitimate visitation or secondary robbing) did not

675 affect bees’ latency to obtain nectar on the first rewarded visit. Boxes indicate the quartiles, and

676 whiskers extend to values within 1.5 times the interquartile range. Letters indicate groups that are

677 significantly different, and sample sizes (number of bees) are given above the boxes 